\title{
EL BIENESTAR UNIVERSITARIO SUBORDINADO A UNA MODERNIDAD INSTRUMENTAL
}

\section{THE UNIVERSITY WELFARE SUBORDINATED TO AN INSTRUMENTAL MODERNITY}

\author{
Luis Felipe Contecha Carrillo1, Luis Guillermo Jaramillo Echeverri²
}

\begin{abstract}
${ }^{1}$ Doctor en Ciencias de la Educación. Área Currículo. Profesor Asociado de la Universidad del Tolima, Cra. 49 sur No. 119 A-108. Ibagué, Tolima Colombia Ifcontecha@iered.org. ${ }^{2}$ Doctor en Ciencias Humanas y Sociales-Educación. Profesor Titular de la Universidad del Cauca, Cra. 3ra. No. 3N-100, Antiguo Liceo. Universidad del Cauca. ljaramillo@unicauca.edu.co
\end{abstract}

Rev. U.D.C.A Act. \& Div. Cient. 14(1): 101 - 109, 2011

\section{RESUMEN}

El presente escrito es una parte de la tesis doctoral Los Sentidos del Bienestar Universitario: más allá de un servicio asistencial. El estudio, permitió comprender, en primera instancia, cómo la Universidad está sumida a patrones de razón instrumental que subordinan el Bienestar Universitario a simple servicio asistencial; en segundo lugar y derivado de lo anterior, al Bienestar Universitario le asiste una mirada utilitarista que se justifica a sí misma como un bien material y, finalmente, frente a esta mirada cosificante de Bienestar, emerge una visión crítica, en especial, por parte de los estudiantes, de concebir el Bienestar Universitario como realización humana, lo cual, va en contravía de principios kantianos de una minoría de edad y un asistencialismo material.

Palabras clave: Vida universitaria, sentidos de bienestar universitario, servicio asistencial.

\section{SUMMARY}

This paper is a part of the doctoral thesis "The Senses of the University Welfare: beyond a welfare service". The study allowed to understand, first, how the University is plunged into patterns of instrumental reasoning, that subordinate the University Welfare to a simple welfare service; secondly and derived from the previous aspect, it was possible to understand how the University Welfare is ruled by a utilitarian look, that justifies itself as a material good; and thirdly, opposite to this objectifying look of Welfare, it emerges the critical vision, especially, on the part of the students, of assuming the University Welfare as human accomplishment, which goes in opposite direction to Kantian principles of dependency and material assistance.

Key words: University life, senses of the university welfare, assistance service.

\section{INTRODUCCIÓN}

El presente artículo, se fundamenta en la Tesis Doctoral "Los Sentidos del Bienestar Universitario: Más allá de un Servicio Asistencial". En ella, se da cuenta de los sentidos que del Bienestar Universitario (BU) tienen los administrativos, los docentes, los funcionarios de las oficinas del bienestar y los estudiantes de tres universidades de la Red de Universidades del Doctorado en Educación en Colombia RUDECOLOMBIA. Para tal fin, fue necesario caracterizar los diferentes momentos históricos del Bienestar, encontrando que el concepto está impregnado por la dinámica de los contextos; esto es, por el desarrollo de acontecimientos políticos, económicos, sociales, educativos, en tanto que algunas de las políticas del Bienestar en la universidad han sido pensadas y diseñadas desde contextos extra universitarios. En consecuencia, el presente escrito cuenta con los insumos de dicha tesis, ampliando los conceptos que de allí se erigieron.

La investigación, se realizó en el contexto de la Educación Superior, identificando, mediante un rastreo histórico y teórico, diferentes momentos, periodos y circunstancias, que moldearon la concepción del Bienestar en la Universidad pública colombiana. En tal sentido, desde el problema de investigación, se logró identificar que el BU está caracterizado 
por: a) la predominancia de una visión tradicional que no consulta las preferencias, la diversidad y la riqueza participativa de sus actores sociales; b) una normatividad que ha generado diversos enfoques conceptuales, los cuales, van desde el Bienestar, como objeto de desarrollo, hasta ser considerado como una política estatal que intenta resolver problemas de pobreza; c) un imaginario de protección estatal hacia las clases menos favorecidas, justificados en la asistencia de un bien material y d) la influencia de experiencias y de modelos de universidades foráneas, espacialmente norteamericanas, en la construcción de las primeras estructuras y estrategias del desarrollo.

El comprender los sentidos del Bienestar en la universidad colombiana, desde los estamentos universitarios, implicó un proceso de reflexión constante, donde cada uno de sus actores sociales (docentes, estudiantes y administrativos), exponían sus sentidos de universidad, academia, espacio universitario y conocimiento; esto permitió comprender que los sentidos acerca del Bienestar, se hallan fuertemente ligados a entes administrativos, los que se amparan bajo la lógica de un Estado benefactor, entendido éste último, como aquel que provee un bien-estar material a los estudiantes, como subsidios de alimentación, de transporte, de vivienda y de becas de estudio, entre otros.

En este sentido, la universidad ha sido pensada a la luz de una formación profesional que engrosa el conocimiento acumulativo de las disciplinas. Los sujetos o actores sociales que hacen parte de la misma, se baten en medio de paradigmas científicos sobre, los que intentan dar solución a enigmas que se plantean como problemas de verdad, en los que, raras veces, se presentan anomalías que pueden dar paso a verdaderas revoluciones científicas (Kuhn, 2001), como bien lo expresara Fuller (1997): la universidad se ha vuelto el guante de tercio pelo que esconde el puño de hierro de la empresa académica.

De esta manera, la producción intelectual de la Universidad está coloreada por el prisma de un desarrollo prometeico de progreso social, en la que se sancionan valores que marcan, al mismo tiempo, el camino y la meta del cambio en las sociedades (Muñoz, 2001). Se puede ver en ella, la intencionalidad de una formación, forjada en el slogan de "cultura ilustrada e intelectual" y que goza aún del prestigio científico, donde sus pensadores son garantes de un conocimiento verosímil e irrefutable, es decir, la universidad aún subsiste bajo principios modernos de razón ilustrada (Escobar, 2004), un lugar para el cultivo de un conocimiento, que se justifica en una formación profesionalizante; espacio donde el hombre se estudia a sí mismo, sin saber, muchas veces, que es fuente y razón de su propio estudio (Foucault, 2001).
Ahora bien, en Colombia, la ley 30 de 1992 (Ley de Educación Superior) normalizó y, a la vez, legalizó, de manera foránea, estas demandas subsidiarias. En tal sentido, la universidad se convierte en una pequeña sociedad en la que su enseñanza se desmarca para dar paso a actividades que le exigen pensar la realidad y la sociedad desde otros lugares de enunciación, ello, unido a demandas académicas que van más allá de la docencia; así, por un lado, se le exige investigar como fuente productora de conocimiento y como política institucional de la academia y, por otro, se encuentra la extensión o la proyección social, como posibilidad para desarrollar estrategias de impacto en la región, conducente a mejorar la calidad de vida de sus habitantes. Estas actividades: docencia, investigación y extensión, hicieron de la universidad un centro de conocimiento sobre, el cual, giran cantidad de sujetos, ya no llamados únicamente docentes, sino también investigadores y gestores comunitarios.

En consecuencia, el rango de acción, la gestión académica y la administrativa de la universidad, la convierte en empresa que debe administrar los recursos que aporta el Estado y aquellos que puede captar con el sector empresarial y gubernamental. Así, la universidad dejó de ser, en su estructura organizacional, un lugar de convivencia entre estudiantes y entre docentes, para convertirse en una institución multifuncional y polifacética, pues no sólo se habla de universidad como centro de enseñanza, también es generadora de conocimiento y una entidad prestadora de servicios.

Se infiere, entonces, una racionalización burocrática de la vida universitaria, lo que implica, que sus administradores piensan en los desarrollos económicos y de producción de sus regiones, a partir de la formación profesional de sus jóvenes. Por ende, los programas de bienestar que se ofrecen al interior de la misma están encaminados, primordialmente, a la dotación de servicios asistenciales, que se decantan en bienes materiales como: servicio de restaurante, subsidios de vivienda (residencias), servicio de biblioteca y becastrabajo, entre otros. El fin asistencial garantiza el logro profesionalizante a alcanzar: la carrera a ejercer y el título a demostrar.

Identificar el BU, como objeto de investigación, implicó una reflexión acerca de los fundamentos teóricos de la producción académica en materia de bienestar universitario. Para ello, fue necesario hacer distinción entre la concepción de producción científica del BU y la producción sobre la calidad, la cobertura, los programas y los servicios. Esta distinción precisó de una clasificación de documentos, dado que hasta el 2003, el ICFES recibió proyectos no investigativos y registró un número considerable de productos relacionados con actividades relacionadas con el bienestar universitario, como 
encuentros, talleres, ponencias e informes de comisiones de trabajo (Contecha, 2009).

En la revisión, se halló que Gutiérrez (1995) escribió el libro "El Bienestar Integral de la comunidad universitaria", conocido como en el primer escrito sobre el tema. Se presenta la evolución del bienestar en la universidad colombiana, en los años anteriores a la publicación de su obra. En el libro "La investigación sobre la Educación Superior en Colombia. Un estado del Arte.", de Martínez de Dueri E Vargas de Avella (2002), analizan 142 informes de investigación, en la que emergieron 11 categorías y, una de ellas, el BU. Dan cuenta de ocho investigaciones ejecutadas por universidades colombianas sobre el bienestar y manifiestan que los estudios que se pueden reconocer como investigaciones y que representan una contribución a la formulación de políticas nacionales son predominantemente contratados por el Estado (ICFES, FONADE, COLCIENCIAS); en ellos, participan reconocidos académicos de las universidades, consultores independientes, representantes de asociaciones y gremios, quienes individualmente o en grupos, avanzan en el estudio de algunas problemáticas. Además, afirman que estos grupos de investigación poseen características de comunidades coyunturales, es decir, el tema de investigación corresponde al interés del organismo que financia el estudio y la permanencia del grupo depende de este interés y del tiempo de duración de los recursos. De los ocho trabajos de investigación sobre el bienestar, siete fueron auspiciados por el ICFES y uno obedece al interés de una universidad.

A pesar del poco interés de las instituciones por la investigación sobre el BU, el libro también da cuenta de un gran número de proyectos sobre alcoholismo y drogadicción en poblaciones universitarias, presentados como trabajos de grado de estudiantes o trabajos aislados, para los programas de especializaciones y que fueron descartados por las autoras en la segunda selección, para constituir la muestra final.

Los antecedentes anteriores justifican una mirada interna a la universidad, desde los sentidos que los actores universitarios otorgan al concepto de Bienestar. El estudio realizado permitió comprender, en primer lugar, cómo la universidad, en su propuesta formativa, se erige bajo parámetros de razón instrumental, a partir de una lógica Kantiana de "minoría de edad"; en segundo lugar, se presentará cómo el concepto de bienestar en la universidad se ha restringido al otorgamiento de un bien material desde una perspectiva utilitarista y, finalmente, se puede apreciar una mirada crítica del BU, especialmente, por parte de los estudiantes, en la que se concibe como acción emancipadora que va más allá de una necesidad.

\section{MATERIALES Y MÉTODOS}

Como método de investigación, se utilizó el enfoque Histórico Hermenéutico, que tiene como fin comprender un fenómeno social con base en la relación que se establece entre diferentes sujetos, a partir de múltiples procesos comunicativos y la comprensión, mediada por la apropiación de la tradición y la historia. Su interés (práctico), se fundamenta en la construcción y en la reconstrucción de identidades socioculturales para, desde esa comprensión, poder sugerir acciones de emancipación (Habermas, 1985). Visión influenciada por otros autores, como Dilthey, Gadamer, Weber y algunos filósofos del lenguaje, como Wittgestein, Searle, Austin y Benveniste, quienes consideran que el comportamiento de los seres humanos no se restringe únicamente a referentes positivistas o cuantitativos.

Si el objetivo de la investigación fue comprender los sentidos que del concepto de bienestar tiene un grupo determinado de personas (estudiantes, profesores y funcionarios universitarios) y si se pretendió desvelar la multiplicidad de realidades, el camino indicado para lograr el objetivo fue aquel donde el mundo depende de las percepciones que el sujeto tenga sobre él, es decir, desde una tendencia subjetiva que trascienda la descripción y se encamine hacia la reflexión, la interpretación y la compresión del BU de la población (Murcia E Jaramillo, 2008).

En esta misma línea de comprensión, el trabajo de campo se inició con tres categorías de análisis a saber: concepto de universidad, sentidos de bienestar y normatividad institucional. En la indagación con los actores sociales fueron emergiendo categorías que sustentaron el estudio, por tanto, el diseño se estructuró con base en el análisis de contenido de documentos institucionales y en las narraciones de los actores universitarios; la herramienta de análisis utilizada correspondió al Atlas. TI. El muestreo teórico utilizado fue el de bola de nieve.

Como unidad de trabajo y unidad de análisis, se seleccionaron tres universidades: Universidad de Caldas, Universidad Pedagógica y Tecnológica de Colombia y Universidad del Tolima, instituciones públicas de educación superior, pertenecientes a la Red de Universidades del Doctorado en Educación RUDECOLOMBIA. Los criterios de selección fueron: ser una universidad miembro de RUDECOLOMBIA; ser universidades de tipo estatal ubicadas en territorios con contextos sociales, educativos, económicos, políticos y culturales; además, que en ellas el BU sea administrado desde diferentes dependencias como vicerrectorías, unidades académicas y, por último, 
ser universidades que tengan estudiantes matriculados en estratos 0-1-2-3 o que cuenten con algún beneficio estudiantil, como becas, subsidios y residencias.

En relación a las técnicas e instrumentos, se realizaron nueve entrevistas en profundidad (Taylor $\mathcal{E}$ Bogdan, 1996), a funcionarios de las oficinas del BU, tres por cada universidad. Además, se ejecutaron 21 sesiones de trabajo, bajo la modalidad de grupos de discusión, según las estrategias utilizadas por Ibáñez (2003); en total, se realizaron 27 sesiones en grupos de discusión y diez entrevistas a profundidad. En cuanto a los aspectos éticos, en cada una de las universidades, donde se desarrolló el trabajo de campo, se solicitó el consentimiento informado de los colaboradores, aclarando que los datos recolectados sería utilizados sólo para fines investigativos.

\section{RESULTADOS Y DISCUSIÓN}

Los presentes hallazgos son parte de la reflexión final del estudio, proceso que sigue a la descripción e interpretación de los datos, por tanto, el énfasis será en la interpretación constitutiva de lo comprendido, una vez se compararon entre sí las categorías logradas.

El BU bajo una mirada adulto-céntrica: un estudiante menor que debe aprender: En la universidad aún subsiste una formación para menores y no necesariamente por la edad de sus estudiantes. Noción de minoría de edad que se refleja en los sentidos de BU por parte de los docentes y los administrativos al considerar que el Bienestar es como el padre o la madre que asiste, materialmente, a los estudiantes con lo que ellos necesitan. "Quiero decir que cuando uno está pequeño tiene un papá y una mamá quienes le brindan a uno, le dan a uno la plata para vestirse, si se enfermó, lo llevan al médico, le enseñan los valores que es lo más importante, usted tiene que comportarse así, y así, y así. Llega un punto en que uno pasa de la adolescencia y ya tiene que empezar a desarrollarse como persona y los papás, de pronto, tiene otras cosas muy diferentes que hacer a las que uno hace $y$, a veces, es complicado, de todas maneras que a uno lo sigan formando ellos. Entonces, cuando uno llega a la Universidad, yo pienso que el bienestar es como el papá de uno, que le dice, mire, yo le presto estos valores, que si se enfermó, mire allá le brindo... bueno todo ese cuento, para concluir, diría: el bienestar es, dentro de la universidad, el papá de los estudiantes" (Administrativo).

Mirada adulto-céntrica que atraviesa los sentidos del bienestar, más por visiones éticas y económicas desde una asistencia adulta que desde el mismo proceso formativo y cognitivo de los estudiantes: El sentido de $\mathrm{BU}$, especialmente, para los docentes, los administrativos y los funcionarios asume al estudiante como el centro del proceso educativo, llegándose a considerar como algo que sólo tiene que ver con el estamento estudiantil:

"A mí se me ocurre otra cosa también, que de pronto en esta universidad, no sé si en todas, porque no las conozco, vemos que hay muchas cosas para los estudiantes, o será que nos estamos contagiando y creemos que lo que le están dando (bien material) a los estudiantes, también nos lo tienen que dar a nosotros, si usted trabaja en cualquier otra entidad como yo trabajé, no hay ningún bienestar" (Administrativo).

El estudiante se desenvuelve en medio de la vida adulta; en ella es visto como alguien que necesita de la universidad, no sólo en asistencia material (alimentación, vivienda y demás), sino también en la formación de valores. Una buena parte de la población entrevistada reconoció que el estudiante debe ser el principal beneficiario de los servicios que ofrece la oficina de Bienestar en la universidad. Expresiones como "estoy pensando que a la Universidad le ha tocado asumir el papel de familia, de alguna manera, de madre regañona, de padre regañón" (docente), dejan entrever que el estudiante aún necesita de un cuidado por parte del mundo adulto en tanto éstos (profesores y administrativos), se sienten con el deber moral de hacerlo. Mirada adulto-céntrica, que considera al estudiante como un ser de necesidades; sentidos de Bienestar, donde el adulto se siente con el deber de decir a los jóvenes universitarios qué valores asumir y cómo se deben comportar. Principio kantiano, que promueve la formación de un sujeto que necesita del experto para desempeñarse con autonomía en la sociedad.

De este modo, el docente no sólo ocupa el papel de transmisor del conocimiento o el administrativo de gestionar los recursos, éstos también se consideran portadores del buen juicio, gracias a que gozan de una visión "madura", capaz de corregir a los y las jóvenes que necesitan de su protección. No en vano, para Kant la ilustración es la salida del hombre de su auto-culpable minoría de edad. Minoría que significa la incapacidad de servirse de su propio entendimiento sin la guía del otro (Kant, 1993). Visión expresada por los funcionarios y los administrativos, quienes ven en los estudiantes un sujeto de necesitad; además del subsidio de alimentación y residencia, sienten que también deben desempeñar el papel de ayudador en la formación moral.

"Es una gran familia, que debe afrontar la formación que no se captó en la casa, donde nosotros recibimos 
la formación primaria, pero cada vez más distante, porque la familia se está desintegrando. Tanto para administradores como para funcionarios, el estudiante debe ser orientado en la norma del buen encauzamiento, de las proporciones de una razón práctica que lo impulsan a ser un hombre bueno, responsable y con buen juicio en la sociedad, debiendo, para ello, hacer uso de la atadura de su propia condición" (menor), que puede ser modelada gracias a una correcta educación. Con la ilustración y el buen encauzamiento, se ingresa a lugares del "deber ser" y "así como debe ser". Se percibe, entonces, un estudiante aprendiz sujeto de obediencia, en tanto las normas propias del comportamiento ético, se encuentran impostadas por una razón adulta.

En tal sentido, el BU adquiere una connotación asistencial de un estudiante febril que necesita de la guía del otro adulto; ahora bien, con lo anterior no se pretende negar que la relación adulto-joven es importante en la convivencia universitaria; no obstante, lo que se someta a crítica es la mirada unilateral por parte del adulto (administrativos, funcionarios del bienestar y docentes) al joven estudiante; pues no se evidenció un principio de reciprocidad por parte de éstos hacia los estudiantes, por el contrario, lo que emergió en los relatos es un estudiante que necesita de cuidado de una comunidad adulta, que se instituye y erige como garante de la formación del estudiantado universitario.

A lo anterior, se suma la concepción de un bienestar enraizado en políticas que hacen ver, principalmente al docente, como agente responsable de la formación del estudiante y no como un sujeto que se puede sentir bien cuando interviene comunicativamente con el estudiantado. Según el informe de la Misión Nacional para la Modernización de la Universidad Pública en 1995, "la dignificación de la docencia se alcanzará cuando los profesores recuperen la conciencia de la importancia y trascendencia de su trabajo y la convicción de su ejemplo, dedicación y permanente formación, determinantes en la formación del estudiante". Se percibe en este informe, un docente dador de Bienestar hacia el estudiante, más no alguien que comparte en relación intersubjetiva con el mismo, por ello, sigue reinando en las políticas educativas una mirada de adulto ayudador, muy cercana a una visión formativa de tipo kantiana.

Kant, en su Tratado de Pedagogía, plantea que los fines de la educación son disciplinar a los hombres, cultivarlos en el obrar de todos los fines posibles, hacerlos prudentes para que puedan vivir en sociedad y moralizarlos para que elijan siempre los fines buenos aceptados por los hombres. "Por cierto, ello impone que la educación busque el endurecimiento de los menores o hacerlos capaces de sobrellevar las contingencia de la vida, para que como adultos todos los seres humanos sean capaces de vivir el deber por el deber", (Kant citado por Vargas Guillen, 2004). La ética en la ilustración, se aferra completamente a los confines de una razón universal, como principio categórico, para el caso específico, un sentido finalista de Bienestar Asistencial.

Esta visión es igualmente compartida por los administrativos, quienes se consideran continuadores y herederos de los padres en la formación de sus hijos; el administrador papá o mamá, siente que debe formar para que puedan estudiar bien, pues a su vez, fueron instruidos con la misma lógica asistencial, cuando eran estudiantes: "Para mí el bienestar tuvo un sentido como estudiante, porque yo fui beneficiario de residencias universitarias, entonces, el sentido era: si mis papás no pueden pagar por la universidad, no pueden pagar por mi sustento, entonces la universidad me ayuda, entonces bienestar, en ese sentido, como un padre para algunos estudiantes" (docente). En suma, el BU refleja sentidos de asistencia material y ética por parte de los adultos a una comunidad estudiantil que asiste a la universidad con sentidos, quizás, distintos a los que consideran los docentes, los funcionarios y los administradores. El sentido de BU adulto se acerca más a un bienestar material, en tanto aplicación moral y económica hacia el estudiante

El bienestar como trayecto que lleva al conocimiento: como posibilidad de realización humana: Este segundo elemento de reflexión y que de manera emergente se hizo presente en el cruce de categorías, son aquellos sentidos que hacen ver el Bienestar Universitario no como fin en sí mismo sino como mediación o trayecto para alcanzar un fin superior. Esta teleología o finalidad es el conocimiento al que se llega para ser profesional, que responde al ideal ilustrado de universidad como productora de conocimiento. Los relatos dejaron entrever cómo los actores universitarios se sienten bien o estánbien, en tanto disfruten de las condiciones necesarias para desarrollar sus funciones en la institución universitaria; por ejemplo: que docentes y estudiantes se encuentren en un ambiente agradable para orientar y recibir clase; de este modo, la universidad sigue siendo entendida como institución profesionalizante, y no como un lugar de enunciación, donde sus actores sociales puedan desarrollar al máximo sus potencialidades humanas. Por el contrario, para éstos, el conocimiento es punto de llegada y el fin último del por qué existe la universidad; la oficina de Bienestar Universitario por su parte, será uno de los canales que hacen posible tal arribo. 
Para la comunidad universitaria que hizo parte del estudio, la universidad es el centro del conocimiento; institución educativa donde el estudiante va a aprender, lugar donde, gracias al conocimiento adquirido, se consigue una carrera y se es profesional. Percepción que también encontraron Murcia et al. (2009), al comprender cómo el imaginario de los estudiantes universitarios se movía en la función de formar como dar forma profesional.

Estos sentidos de universidad hacen del Bienestar subsidiario de la profesión, en tanto el conocimiento ocupa un lugar central en la formación del estudiante. El Bienestar pasa a ser el trayecto o camino por el que circula, no la formación, sino la profesión a alcanzar; de este modo, se logra que el estudiante se forme profesionalmente sin mayores preocupaciones; "bienestar son las condiciones que permiten estar bien dentro de la universidad, que tanto el estudiante como el profesor y el empleado no tengan mucho de qué preocuparse dentro de la universidad, entonces, tenemos programas que son servicios de salud" (docente).

Se deja entrever, entonces, una mirada utilitarista del Bienestar, en la que no se valoran las acciones de los sujetos como seres en situación sino los estados de los espacios y escenarios para que éstos se sientan bien, lo cual, lleva a una des-personalización del sujeto y, por ende, la pérdida de valor de las intenciones y los motivos del universitario a la hora de evaluar las acciones. Se percibe una reducción de los medios para llegar a los fines, es decir, el BU es importante en tanto prevé que los actores sociales estén en condiciones de llegar al tan anhelado conocimiento profesionalizante. Se llega, por tanto, a un consecuencialismo adverso que puede responsabilizar al Bienestar si los actores universitarios no alcanzan la meta del conocimiento.

Comparar el Bienestar con el estado de los espacios universitarios y los programas sociales que se realizan como garantía para llegar a un conocimiento intelectual, implica reducirlo a mediación y certeza fugaz; es colocar la vida misma de los sujetos en torno a una intelectualidad profesionalizante. Bajo esta perspectiva, se encontró que las tres oficinas de bienestar, que hicieron parte del estudio, justifican sus servicios en acciones como: el servicio de restaurante, el subsidio de vivienda, la adecuación de salones y de pasillos, programas de cultura, deporte y recreación, todas ellas, como garantes de una universidad que busca la excelencia académica del conocer, tal y como lo consideró un administrativo: "para mí el Bienestar es un área de conocimiento donde uno se puede formar desde la parte académica hasta la parte cultural, hasta la parte deportiva $y$ puede aprender muchísimas cosas, por eso digo que es un área del conocimiento". Bienestar que se justifica por las atenciones que pueda ofrecer a la comunidad universitaria; sin embargo, pasa a ser subsidiario del fin último universitario: el conocimiento. Incluso, los funcionarios de la oficina de bienestar reconocen que lo que se muestra como gestión del bien-estar en su dependencia administrativa son sólo cifras estadísticas que permiten respaldar su labor frente a los procesos de acreditación institucional.

Esta visión reduccionista, se adhiere a propuestas teóricas que terminan colocando el Bienestar como mediación funcional para alcanzar el conocer. "La formación académica y la formación ética, el desarrollo de la sensibilidad y el impulso al deseo y a la voluntad del saber son, desde esta perspectiva, las tareas centrales de un bienestar universitario, que debe ir mucho más allá de la satisfacción de las necesidades materiales más inmediatas" (Hernández, 2002). Si bien la intención del autor es desbordar lo material, inevitablemente cae en él, por cuanto el fin material es lograr el tan apreciado conocimiento, a través de una profesión certificada mediante un título.

Asumir el bienestar desde una mirada crítica implicará descentrar el conocimiento, como fin último, para empezar a buscar otras rutas que le permitan a la comunidad universitaria asumirlo como capacidad humana y no como trayecto de conocimiento. Llegará el momento donde la Universidad dejará de formar a sus estudiantes para una vida profesional como seres autómatas y empezará a considerarlos como sujetos de potencia, que otorgan vida y significado a lo que hacen. Será en ese momento cuando el BU dejará de ser trayecto para el conocer y se convertirá, al fin y al cabo, en fuente de realización humana, que trasciende el concepto pírrico de bienestar como beneficencia material y ven-eficiencia intelectual.

El bienestar como capacidad: la visibilidad del ser: Sen (1999), a partir de una crítica extensa que hace al concepto de bienestar, dice que este se ha reducido a la mirada económica y, por ende, sólo se constriñe a fines utilitaristas, que se representa en bienes materiales; en contraposición a esta reducción, propone asumirlo como potencia, es decir, como la posibilidad que tiene el sujeto de realizarse gracias a sus capacidades humanas; concibe el bienestar en términos de lo que una persona puede "realizar", logrando un sentido de actividades, como estados de existencia o del ser que, a su vez, se convierte en un vector de realizaciones. Unido a esto, asume al ser no como sujeto sino como agente, en tanto es alguien 
que puede decidir y actuar con libertad (Nussbaum E Sen, 2002).

Esta asunción del bienestar, se comprendió en las expresiones de los estudiantes, quienes reclaman ser tenidos en cuenta, no sólo por aquello que se les pueda dar asistencialmente, sino también por lo que puedan opinar, decir, hacer y debatir en medio de la universidad. Lo que subyace en sus voces es un reclamo de visibilidad, como agentes de cambio y no sólo como usuarios de un servicio. "Pues bienestar, yo creo que también es permitir el espacio para la confrontación de ideas, no sólo físico sino intelectual porque aquí es necesario remitirse a los que han venido presentándose en la universidad, se estableció un debate en cuanto a lo que no se está de acuerdo del estatuto general de la universidad, se llegó a vías de hecho por falta de comprensión y de escucha, por falta del Consejo Superior Universitario (...). Lo que se debe tener en cuenta es la opinión del estudiante, de tener en cuenta la opinión de la comunidad universitaria, eso es bienestar y, además, que genera sentido de pertenencia, pues de lo que estoy hablando es importante" (estudiantes).

Más allá del bien material utilitario, se propugna la voz de un estudiante que busca espacios donde se confronten sus ideas, se piense la universidad y sea tenido en cuenta respecto a las decisiones que toman las directivas universitarias; en su voz, se escucha el no ser mirado como menor en edad y en pensamiento, tampoco como alguien que simplemente aprende, es más bien agente y constructor de un lugar que siente como propio, llamado universidad; pues lo que él dice como agente es también importante en el mundo de la vida universitaria.

Para el estudiante, su palabra tiene sentido si es escuchada y validada como importante para el concierto académico. No desea ser usuario de un servicio académico; por el contrario, reclama sentirse acogido y asumido en la reciprocidad de una voz que tiene algo que decir en los claustros universitarios. "Bienestar no es solamente academia, sino que depende de otras cosas, lo que yo si creo, es que en esta universidad y en muchas universidades, se ha enfocado más a lo que no debe ser o a lo que está en segundo plano, a lo que debe ser como tal es el bienestar académico que se defiende sobre cualquier cosa es fundamental, aunque no sólo para fortalecer la academia. Lo que sí creo que tampoco se justifiquen a eso y otras cosas que permiten que el estudiante se incentive, eso es fundamental" (estudiante).

Lo anterior proyecta el Bienestar como mediación para instalarlo como fuente de realización de la vida misma; como apertura enunciativa que hace al estudiante sentirse bien, pues no se considera un menor que, potencialmente, llegará a ser adulto. Por el contrario, es agente que puede participar de la vida universitaria con los deberes y los derechos que le asisten. Ser tenido en cuenta es ser escuchado como alguien que puede tener la razón, que su voz no es acallada por ser visto por el mundo adulto como inmaduro o viche; quiere ser asumido como válido al interior de esos contratos de habla que le hacen ser hablante activo al interior de una conversación. En palabras de Sen (1999), como alguien que tiene igualdad de oportunidades para el Bienestar.

Por último, es de resaltar que no se hace referencia a un Bienestar donde sólo se escucha la voz del estudiantes, sino un bienestar para todos los actores sociales que hacen parte del mundo universitario; un lugar donde todos puedan participar, debatir y reflexionar la universidad que desean -no sólo que necesitan-, en principios de reciprocidad y acción comunicativa, en la que ninguno preste sus labios para que otros hablen por ellos, ni sean anónimos o terceros en la vida universitaria; un bienestar donde la normativa institucional sea debatida y construida en interacción permanente. Se aboga, entonces, por un Bienestar que encuentra eco en la virtud y la realización, un lugar donde la norma sea confrontada de manera dialógica; no obstante, no es la norma o deber Kantiano asumido por sólo desde la razón, es más bien la norma aristotélica que encuentra sentido en su realización, pues no se hace por temor al castigo o la penalización. "En Aristóteles, la virtud es lo que constituye la vida completa; es la condición necesaria para la mejor vida posible. Cualquier otra forma de vida no alcanzaría la norma de la vida humana" (Hartnack, 1994). Es posibilidad de acción sin imposición y sometimiento, la cual, lleva en sí misma el gusto de la realización que conduce a una vida plena sin reducciones y sin esclavismos impuestos para sí mismos o para otros. Este es el bienestar universitario que, tal vez, reclama el estudiante y que tiene poca repercusión en la vida adulta de los administrativos y de los docentes.

Concebir el bienestar universitario más allá de un asistencialismo adulto, que permite alcanzar conocimiento, es reconocer que aún seguimos enclaustrados en una modernidad que muestra una sola vía de conocimiento, donde aún unos se erigen como portadores absolutos del conocer y, otros, como recipientes que aprenden, con el fin de alcanzar un título. Aún suena imposible concebir el BU más allá de una razón instrumental que forma profesionales para un futuro poco promisorio; queda como reto empezar a asumir el Bienestar en nuestras universidades como capacidad, como hospitalidad y 
acogida donde el otro pueda hablar y dejar de ser un anónimo más en su comunidad universitaria; ser al fin otro que no sólo piensa para existir, sino que puede (capacidad) para ser.

Agradecimiento: Los autores agradecen la lectura crítica por parte de los evaluadores, así como sus pertinentes observaciones. De igual manera, damos crédito a la Universidad del Tolima y Universidad del Cauca, en la construcción del presente documento. Conflicto de intereses: El manuscrito fue preparado y revisado con la participación de todos los autores, quienes declaramos que no existe ningún conflicto de intereses que ponga en riesgo la validez de los resultados presentados.

\section{BIBLIOGRAFÍA}

1. CONTECHA, L.F. 2009. Un Estado del arte del bienestar universitario en Colombia. Revista Perspectivas Educativas. Maestría en Educación. Universidad del Tolima, Ibagué, Colombia. 2:109-127.

2. ESCOBAR, A. 2004. Desplazamiento, desarrollo y modernidad en el pacífico colombiano. En: Restrepo, E.; Rojas, A. (eds.) Conflicto e (in)visibilidad. Retos en los estudios de la gente negra en Colombia. Popayán: Universidad del Cauca. p.53-72.

3. FOUCAULT, M. 2001. Las palabras y las cosas. Siglo XXI Editores. (Buenos Aires). 378p.

4. FULLER, S. 1997. La comprensión pública de la ciencia: nuestro último pánico moral. Trad. Dalia Restrepo (2002). Seminario de Doctorado en Ciencias Sociales, Niñez y Juventud: Universidad de Manizales - CINDE. p.1-39.

5. GUTIÉRREZ, A. 1995. El bienestar integral de la comunidad universitaria. Universidad Javeriana. Bogotá.

6. HABERMAS, J. 1985. Ciencias sociales reconstructivas vs. Ciencias comprensivas. En: Ciencia moral y acción comunicativa. Ed. Pensament (Barcelona). 30p.

7. HARTNACK, J. 1994. Breve historia de la filosofía. Ed. Teorema (Madrid). 260p.

8. HERNÁNDEZ, C. 2002. Universidad y Excelencia. Instituto Colombiano para el Desarrollo de la Ciencia y la Tecnología "Francisco José de Caldas" y Asociación Colombiana de Universidades. En: Educación Superior. Sociedad y Educación. (Bogotá). p.19-148.

9. IBÁÑ̃E, J. 2003. Más allá de la sociología. El grupo de discusión: técnica y crítica. Ed. Siglo Veintiuno (Madrid). 428p.

10. KANT, I. 1993. Respuesta a la pregunta: ¿̇qué es la ilustración? Ed. Tecnos. (Madrid). 180p.

11 KUHN, T.S. 2001. La estructura de las revoluciones científicas. Fondo de Cultura Económica. (México). 128p.

12. LEY 30 DE 1992 (Diciembre 28) "Por la cual se organiza el servicio público de la Educación superior". En Diario Oficial 40700 de Diciembre 29 de 1992.

13. MARTÍNEZ DE DUERI, E.; VARGAS DE AVELLA, M. 2002. La investigación sobre Educación Superior en Colombia. Un estado del Arte. Serie Calidad de la Educación Superior. ICFES. 123p.

14. MISIÓN NACIONAL PARA LA MODERNIZACIÓN DE LA UNIVERSIDAD PÚBLICA. Informe Final. 1995. Ed. Presencia Limitada (Bogotá). 166p.

15. MUÑOZ, G. 2001. Notas al concepto de desarrollo. Centro Internacional en Niñez y Juventud. Universidad de Manizales. (Manizales). 26p.

16. MURCIA, N.; PINTOS, J.L.; OSPINA H., F. 2009. Función versus institución: imaginarios de profesores y estudiantes universitarios. Educación y Educadores. 12(1):63-91.

17. MURCIA, N.; JARAMILLO, L. 2008. La complementariedad etnográfica. Investigación Cualitativa. Una guía para abordar estudios sociales. Ed. Kinesis (Armenia). 228p.

18. NUSSBAUM, M.; SEN, A. 2002. La calidad de vida. Ed. Fondo de Cultura Económica (México). 588p.

19. SEN, A. 1999. El futuro del Estado de Bienestar. La factoría, 8. Disponible desde Internet en: http:// www.lafactoriaweb.com/articulos/amartya.htm. (con acceso 12/06/07).

20. TAYLOR, S.; BOGDAN, R. 1996. Introducción a los métodos cualitativos de investigación. Ed. Paidós (Barcelona). 434p. 
21. VARGAS, G. 2004. Kant y la pedagogía: fenomenología de la génesis individual y colectiva del imperativo moral. Coloquio Internacional sobre Currículo, 3. Memorias. Popayán. Universidad del Cauca. 18p.

Recibido: Julio 28 de 2010

Aceptado: Marzo 7 de 2011 